\title{
Effects of Cornus Officinal Is Total Glycoside on Cardiomyocyte Apoptosis and Calcium Overload after Hypoxia/Reoxygenation Injury in Rats
}

\author{
Ke Chen*, Liudan Chen*, Qihui Huang, Aizhen Pan, Kefang Chen, Xiangping Hou, \\ Xiaojin Xue, Jianjun Li ${ }^{\#}$ \\ Department of Traditional Chinese Medicine, Sun Yat-sen Memorial Hospital Affiliated to Sun Yat-sen University, \\ Guangzhou, China \\ Email: ${ }^{*}$ Ljj1965wuan@126.com
}

How to cite this paper: Chen, K., Chen, L.D., Huang, Q.H., Pan, A.Z., Chen, K.F., Hou, X.P., Xue, X.J. and Li, J.J. (2019) Effects of Cornus Officinal Is Total Glycoside on Cardiomyocyte Apoptosis and Calcium Overload after Hypoxia/Reoxygenation Injury in Rats. Chinese Medicine, 10, 1-10. https://doi.org/10.4236/cm.2019.101001

Received: December 21, 2018

Accepted: January 27, 2019

Published: January 30, 2019

\section{Copyright $\odot 2019$ by author(s) and} Scientific Research Publishing Inc. This work is licensed under the Creative Commons Attribution International License (CC BY 4.0).

http://creativecommons.org/licenses/by/4.0/

(c) (i) Open Access

\begin{abstract}
Objective: To investigate the mechanism of Cornus officinalis Total Glycosides (COTG) on myocardial protection, by studying effects of COTG on cardiomyocyte apoptosis induced by hypoxia/reoxygenation and calcium concentration in rats. Methods: The myocardial cells of born 1-3d SD rats were isolated by enzyme digestion, cultured for 3 days. Cells were divided into five groups: Control group, H/R group, Cornus officinalis Total Glycosides low-dose group (LDG), Cornus officinalis Total Glycosides middle-dose group (MDG) and Cornus officinalis Total Glycosides high-dose group (HDG). Three drug groups were pretreated with different doses of Cornus officinalis Total Glycosides before hypoxia/reoxygenation treatment. The apoptotic rate was determined by flow cytometry assay, the intracellular free calcium concentration was examined by flow cytometry, and the ultrastructure of myocardial cells was observed under transmission electron microscope. Results: The results revealed that Cornus officinalis Total Glycosides pretreatment decreased apoptosis rate, but the effect of lower dosage is not significant. Furthermore, Cornus officinalis Total Glycosides can attenuate mitochondrial calcium overload, improve mitochondrial morphology and inhibit cardiomyocyte apoptosis caused by H/R. Conclusion: Cornus officinalis Total Glycosides pretreatment can inhibit cardiomyocyte apoptosis and calcium overload during H/R injury. However, the underlying mechanisms require us to further study.
\end{abstract}

\section{Keywords}

Cornus officinalis Total Glycoside, Hypoxia/Reoxygenation, 
Cardiomyocyte Apoptosis, Calcium Overload

\section{Introduction}

Currently, ischemia heart disease is an overwhelming health problem worldwide [1] [2]. Ischemia/reperfusion (I/R) injury is the phenomenon that ventricular remodeling and cardiac dysfunction are aggravated, after ischemic myocardium restores blood supply. I/R injury can lead to the changes of myocardial structure and function, then systolic and diastolic dysfunction and lipid metabolism disorder, which further induce reactive oxygen species and cardiomyocyte apoptosis [3]. Cardiomyocyte apoptosis includes two major pathways, the extrinsic pathway via death receptors, and the intrinsic pathway involving mitochondria [4] [5].

Numerous studies have confirmed that calcium overload plays a key role in myocardial apoptosis induced by myocardial $\mathrm{I} / \mathrm{R}$ injury [6]. Cornus officinalis Sieb. et Zucc., a kind of traditional Chinese herb, has effect in nourishing liver and kidney and astringent. Cornus officinalis Total Glycosides are the main active components of Cornus officinalis Sieb. et Zucc., which have anti-inflammatory effects [7]. Our previous studies have been proved that Cornus officinalis Total Glycosides can improve myocardial ischemia injury by promoting mitochondrial biogenesis [8]. Nevertheless, it is unclear about the effect of Cornus officinalis Total Glycosides on myocardial ultrastructure and calcium concentration after ischemia/reperfusion injury. To mimic cardiomyocyte I/R injury, we established a hypoxia/reoxygenation $(\mathrm{H} / \mathrm{R})$ model in myocardial cell. In this study, we investigated the protective effect of Cornus officinalis Total Glycosides in cardiomyocytes against H/R-induced apoptosis and whether the mechanism involved inhibition calcium overload.

\section{Materials and Instruments}

\subsection{Experimental Animals}

Sprague-Dawley rats of SPF level, 1 to 3 days, weighing $20-30 \mathrm{~g}$, were provided by the Animal Experimental Center of Sun Yat-sen University (number of animal license SCXK 2011-0029).

\subsection{Reagents and Drugs}

Cornus officinalis Total Glycosides were provided by Guangdong Research Institute of traditional Chinese Medicine.

Main reagents: DMEM/F12 culture-medium (Gibco); DMEM sugar-free culture-medium (Gibco); Fetal bovine serum (FBS, Gibco); 0.25\% Trypsin (Gibco); Penicillin-streptomycin solution (Gibco); SABC anti $\alpha$-Sarcomeric actin kit (Boster Biological Technology Co. Ltd.); Fluo-4/AM (Biyuntian Biological Technology Co. Ltd.); Annexin V-FITC kit (eBioscience); 2.5\% Glutaraldehyde 
and 2\% Paraformaldehyde (Guangzhou Ray Biotech Co. Ltd.).

\subsection{Instruments}

Micro sampler and $\mathrm{CO}_{2}$ incubator (Thermo); 3K15 desktop high-speed refrigerated centrifuge machines (Sigma); Super clean bench (Airtech); Fluorescence microscope (Nikon); Electron microscope (FEI); Flow cytometry (BD); Electronic balance (Sartorius); Filter (Millipore); 6 hole cell culture plate (Nunc); 6 $\mathrm{cm}$ Petri dish and centrifuge tube (Corning).

\section{Methods}

\subsection{Primary Culture of Myocardial Cells}

Improving the method reference to literatures [9] [10], the $1-3$ days of SD rats were soaked in $70 \%$ alcohol for disinfection; then myocardial cells were isolated by enzyme digestion, which were transferred into the complete medium (CM ingredients: DMEM-HG $500 \mathrm{ml}, 10 \%$ fetal bovine serum $50 \mathrm{ml}$, penicillin-streptomycin solution $5 \mathrm{ml}$ ) to make single cell suspension. After fibroblasts were removed by differential velocity adherent technique, cells were cultured in diverse culture dishes with humidified air $\left(5 \% \mathrm{CO}_{2}\right)$ at $37^{\circ} \mathrm{C}$; Cells were seeded in six-well plates $\left(2-5 \times 10^{5}\right)$. The medium was changed every 24 hours.

\subsection{Identification of Primary Myocardial Cells}

Identification of myocardial cells by immunohistochemistry method, specific procedures reference the instructions of SABC anti $\alpha$-Sarcomeric actin kit. The expression of actin in myocardium was observed, and the positive rate of myocardial cells was calculated.

\subsection{Experimental Grouping and Establishment of H/R Model}

The isolated primary myocardial cells were divided into five groups: 1) Control group: Cells were cultured under normal conditions. 2) H/R group: To establish the hypoxia condition of the H/R model, after 3 days of normal culture, cells were subjected to hypoxia using glucose-free and FBS-free DMEM/F12 buffer that surface were sealed with sterile liquid paraffin, which were cultured in a hypoxia chamber suffused with $5 \% \mathrm{CO}_{2}$ and $95 \% \mathrm{~N}_{2} 2$ hours. Cells were then transferred to $10 \%$ FBS DMEM/F12 in normal conditions for reoxygenation 24 or 48 hours. 3) Cornus officinalis Total Glycosides low-dose group (LDG): Cells were pretreated with $0.0049 \mathrm{~g} / \mathrm{l}$ Cornus officinalis Total Glycosides for 24 hours and then were subjected to hypoxia/reoxygenation as H/R group. 4) Cornus officinalis Total Glycosides middle-dose group (MDG): Cells were pretreated with $0.049 \mathrm{~g} / \mathrm{l}$ Cornus officinalis Total Glycosides for 24 hours and then were subjected to H/R. 5) Cornus officinalis Total Glycosides high-dose group (HDG): Cells were pretreated with $0.49 \mathrm{~g} / \mathrm{l}$ Cornus officinalis Total Glycosides for 24 hours and then were subjected to H/R. 


\subsection{Determination of Myocardial Apoptosis Ratio}

Using flow cytometry assay. Following successful establishment of cardiomyocyte H/R injury model and different treatments, cells of each group were collected and resuspended at a density of $1 \times 10^{6} / \mathrm{ml}$. The Annexin V/PI of apoptotic cells were counted, specifically as follows: supernatant was abandoned, all operations are strictly operated according to Annexin V-FITC kit instructions. Cells were incubated with propidium iodide and annexin $\mathrm{V}$-fluorescein isothiocyanate for $15 \mathrm{~min}$ in the dark; under the $488 \mathrm{~nm}$ excitation light, the apoptotic rate was determined by flow cytometry. The above experiment was repeated three times.

\subsection{Examination of Intracellular Free Calcium Concentration $\left(\left[\mathrm{Ca}^{2+}\right] \mathrm{I}\right)$ in Cardiomyocytes}

Cells were seeded in 96-well plates under serum-free culture conditions for 24 hours, were washed with HBSS solution 3 times, were added Fluo-4/AM working fluid and were incubated for $30 \mathrm{~min}$ in the dark at $37^{\circ} \mathrm{C}$ thermostat. Next, cells were washed with HBSS solution 3 times to fully remove Fluo-4/AM working fluid. Finally, using flow cytometry to examine intracellular free calcium concentration, the excitation wavelength was $494 \mathrm{~nm}$ and the emission wavelength was $516 \mathrm{~nm}$.

\subsection{Ultrastructure of Myocardial Cells under Transmission Electron Microscope}

After the corresponding treatment, cells of each group were washed, digested, centrifugalized, collected, and fixed by fixation fluid (2.5\% Glutaraldehyde $+2 \%$ Paraformaldehyde). Following a series of acetone dehydration ( $70 \%$ acetone for $15 \mathrm{~min}, 80 \%$ acetone for $15 \mathrm{~min}, 90 \%$ acetone for $15 \mathrm{~min}$, and $100 \%$ acetone for $10 \mathrm{~min}$ ), cells were embedded in rubber. Then embedded block were cut into sections of 1 to $10 \mu \mathrm{m}$ by microtome and sections were stained with dye liquor. Lastly, the ultrastructure of myocardial cells was observed under transmission electron microscope.

\subsection{Statistical Analysis}

SPSS 13.0 statistical software was used for statistical analysis. The experimental data were expressed as means \pm standard deviation (SD) and analyzed by ANOVA and paired $t$ test. $\mathrm{P}<0.05$ was considered statistically significant.

\section{Results}

\subsection{Morphological Observation of Primary Myocardial Cells}

Myocardial cells were observed under inverted microscope. Newly inoculated cells were suspended in the culture medium, round and bright. After 24 hours of culture (Figure 1), most cells attached to the wall, gradually extended pseudopodia and became to polygon shape. The nucleus was small and round. Even it 


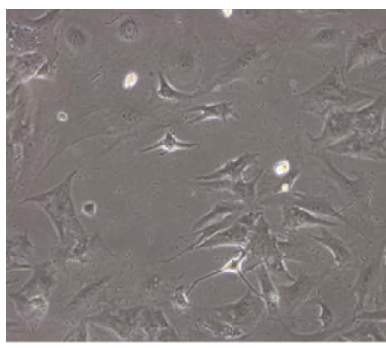

(a)

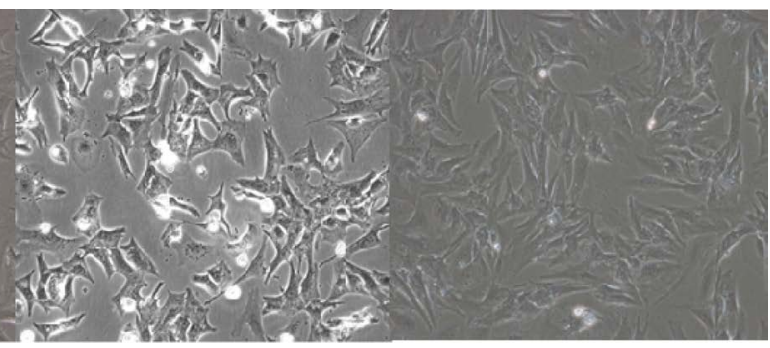

(b)

(c)

Figure 1. Primary cultured rats myocardial cell $(\times 200)$. (a) Primary culture 24 h; (b) Primary culture 48 h; (c) Primary culture 72 h.

can occasionally be seen that contraction of a single cell, however the pulse amplitude was small, speed was an average of 20 - 30 times/minute. After cultured for $48 \mathrm{~h}$ (Figure 1 and Figure 2), cells were found gathering closely and grew over all the bottom. It was can be seen that more than $90 \%$ of the cells begun to pulse at a frequency of $60-80$ times/min. The primary cells fused into pieces and formed a monolayer after cultured for $72 \mathrm{~h}$ (Figure 1), which pulse frequency is $110-130$ times/min.

\subsection{Identification of Primary Myocardial Cells (Figure 2)}

Myocardial cells contain $\alpha$-Sarcomeric actin [11] and we chose SABC anti $\alpha$-Sarcomeric actin as the first antibody in this study. Therefore, the cytoplasm of myocardial cells should be dark brown after staining. After repeated three calculations, the positive rate of myocardial cells was $96.32 \%, 97.81 \%$ and $96.95 \%$. The average value was $97.027 \%$, which is purity of myocardial cells.

\subsection{Effects of Cornus officinalis Total Glycoside on the Apoptosis Rates of Rat Cardiomyocytes Induced by H/R (Figure 3)}

The apoptosis rates of cardiomyocytes were tested by flow cytometry of Annexin-V/PI double staining. As shown in Figure 3, the apoptosis rate in the control group was lower than that of the H/R group, Cornus officinalis Total Glycosides low-dose group, middle-dose group and high-dose group $(\mathrm{P}<0.01)$; compared with the H/R group, the apoptosis rates in middle-dose group and high-dose group was significantly decreased, the difference was statistically significant $(\mathrm{P}<$ $0.05)$. However, there was no statistically significant difference between low-dose group and H/R group. Above results suggested that Cornus officinalis Total Glycosides, mainly middle-dose and high-dose, could exert cardioprotective effects by inhibiting H/R-induced apoptosis of myocardial cells.

\subsection{Effects of Cornus officinalis Total Glycoside on Intracellular Free Calcium Concentration ([Ca $\left.{ }^{2+}\right]$ I) (Figure 4)}

In this study, Fluo4-am probes were used to label myocardial cells and intracellular free calcium concentration $\left(\left[\mathrm{Ca}^{2+}\right] \mathrm{I}\right)$ was detected by flow cytometry. In the $\mathrm{H} / \mathrm{R}$ group, the relative fluorescence intensity of $\mathrm{Ca}^{2+}$ was about 340 , which 


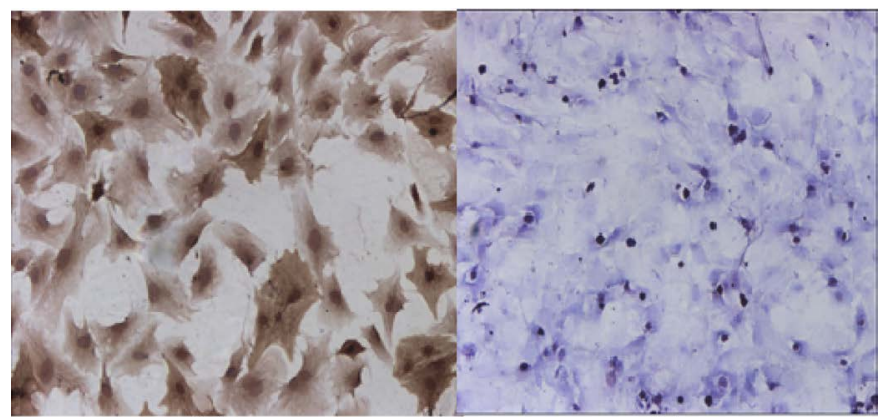

(a)

(b)

Figure 2. Immunohistochemical staining of neonatal rat cardiomyocytes $(\times 200)$. (a) Myocardial cells with positive anti $\alpha$-Sarcomeric actin staining; (b) Negative control (Distilled water instead of first antibody).

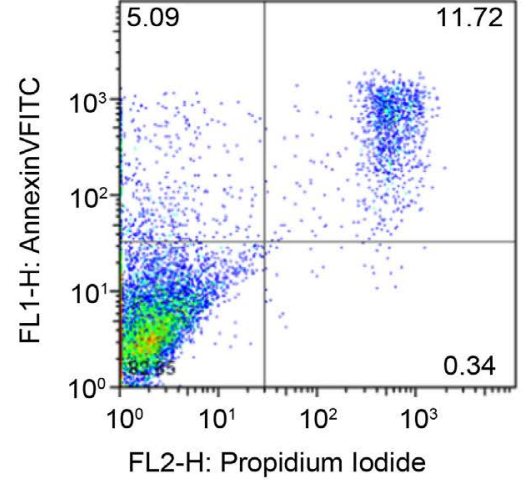

(a)

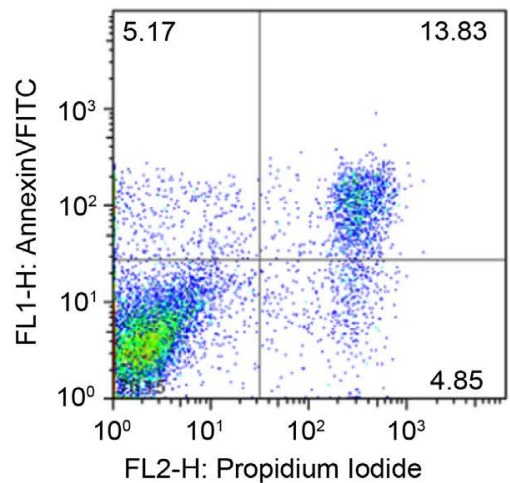

(d)

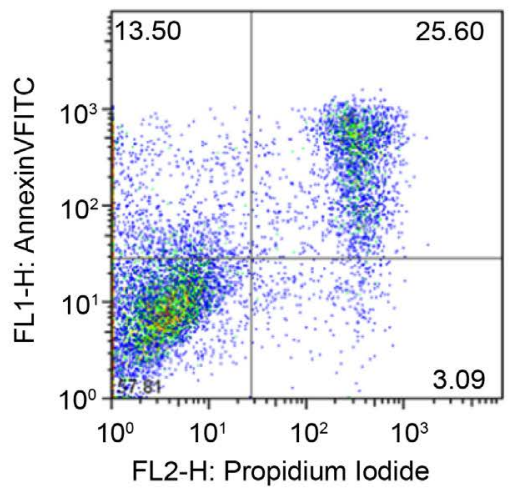

(b)

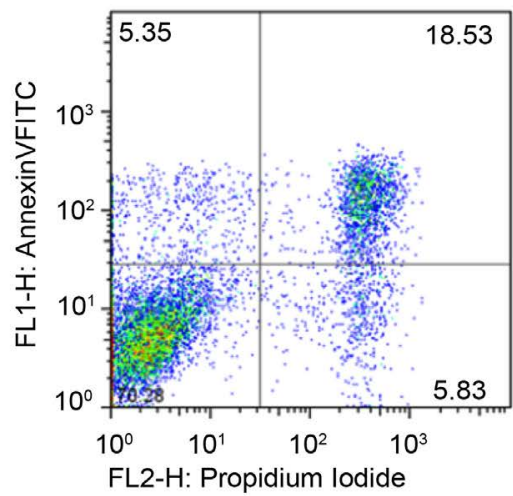

(e)

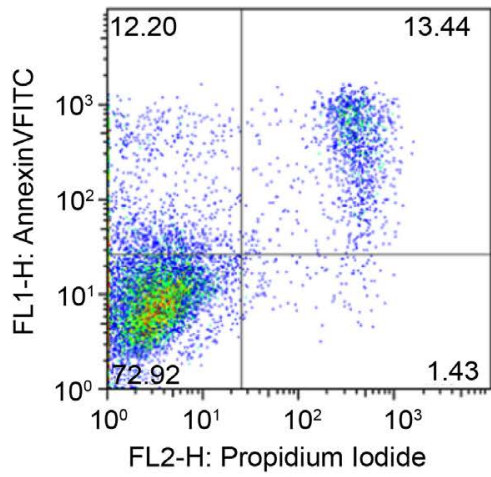

(c)

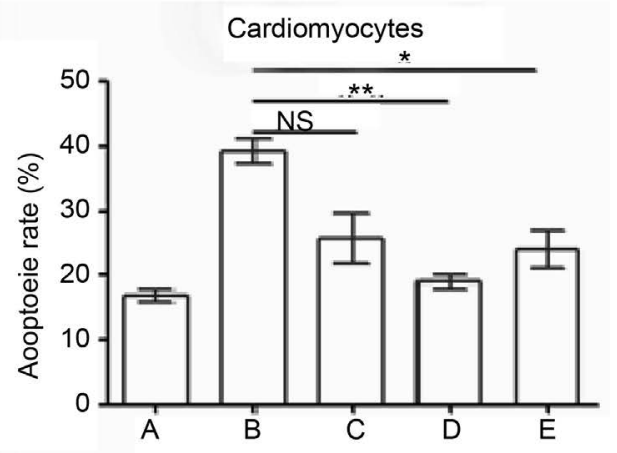

(f)

Figure 3. Effects of Cornus officinalis Total Glycoside on the apoptosis rates of rat cardiomyocytes in five groups. (a)-(e) Typical images evaluated by flow cytometry; (f) The apoptosis rates was quantified by BD FACS software; (a) Control group; (b) H/R group; (c) Cornus officinalis Total Glycosides low-dose group; (d) Middle-dose group; (e) High-dose group. Values were expressed as mean $\pm \mathrm{SD} .{ }^{\star} \mathrm{P}<0.01$, vs control group; ${ }^{\star *} \mathrm{P}<0.01$, vs $\mathrm{H} / \mathrm{R}$ group.

strengthened markedly compared with control group. The relative fluorescence intensity of Cornus officinalis Total Glycosides low-dose group, middle-dose group and high-dose group were approximately 330, 110 and 200. Compared to the $H / R$ group, fluorescence intensity of middle-dose group and high-dose group decreased significantly $(\mathrm{P}<0.01, \mathrm{P}<0.05)$, indicating that pretreatment 


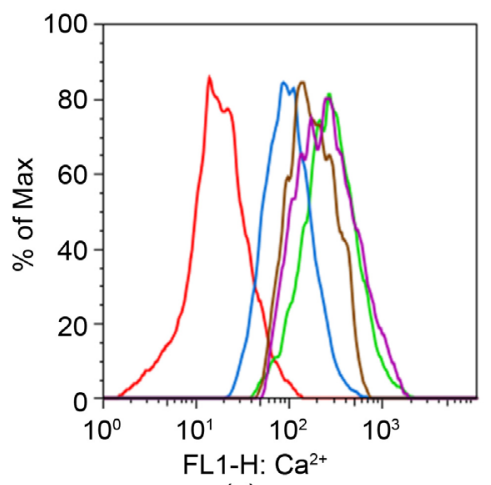

(a)

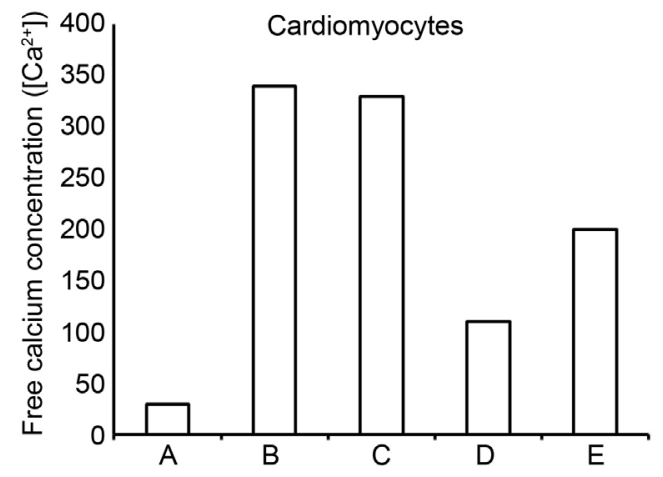

(b)

Figure 4. Effects of Cornus officinalis Total Glycoside on intracellular free calcium concentration after H/R injury. A, Control group; B, H/R group; C, Low-dose group; D, Middle-dose group; E, High-dose group. Values were expressed as mean $\pm \mathrm{SD}$. ${ }^{\star} \mathrm{P}<0.01$, vs control group; ${ }^{*} \mathrm{P}<0.01$, vs $\mathrm{H} / \mathrm{R}$ group.

with middle-dose and high-dose of Cornus officinalis Total Glycosides can block H/R-induced calcium overload of myocardial cells. There was no significant difference between low-dose group and H/R group, which suggested that intervention of low-dose Cornus officinalis Total Glycosides had no effect on H/R-induced calcium overload.

\subsection{Effects of Cornus officinalis Total Glycoside on Myocardial Mitochondria Ultrastructure after H/R Injury (Figure 5)}

Mitochondrial morphological changes were observed by the electron microscope. The ultrastructure of myocardial cells was not abnormal in control group, such as loose nuclear chromatin, uniform density, complete membrane of mitochondria, dense crista, etc. In contrast, while changes in mitochondrial morphology, including disorganization, swelling, and reduction of the crista, were increased. After pretreatment of Cornus officinalis Total Glycosides, the degree of myocardial injury induced by $\mathrm{H} / \mathrm{R}$ was significantly weakened and the mitochondrial membrane was intact in middle-dose and high-dose group. Whereas, the damage of myocardial mitochondria caused by $H / R$ was not significantly improved in low-dose group.

\section{Discussion}

Our previous studies revealed that Cornus officinalis Total Glycosides, a promising cardioprotective Chinese herbs extract, inhibit cardiomyocyte apoptosis of suckling mouse in vitro [12] and improve myocardial ischemia injury by promoting mitochondrial biogenesis [8]. Mitochondria are important organelles for the energy supply of cardiac myocytes. Myocardial ischemia reperfusion injury is common in clinic and its mechanism is complex. I/R is closely related to cardiomyocyte apoptosis, calcium overload and release of inflammatory mediators [13]. And many injury factors including $\mathrm{H} / \mathrm{R}$ injury can induce apoptosis by activating death receptor dependent signal transduction pathway and mitochondrial 


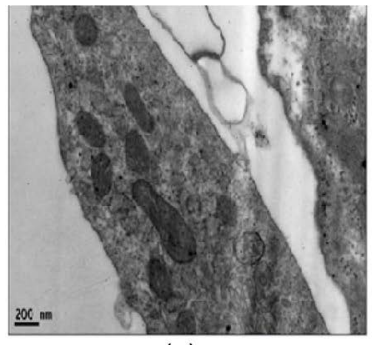

(a)

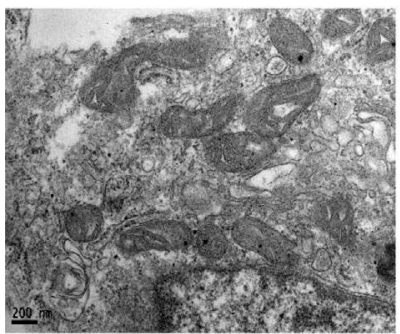

(d)

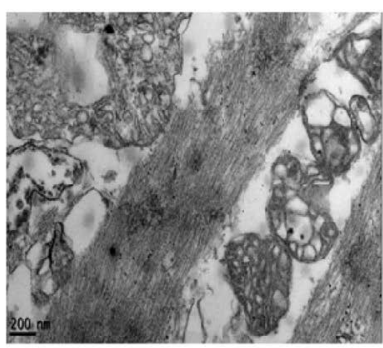

(b)

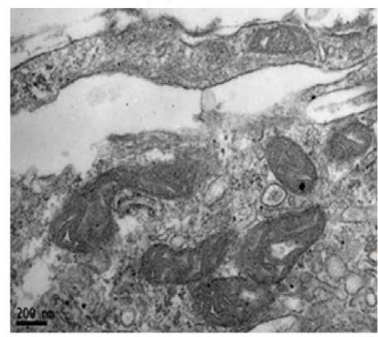

(e)

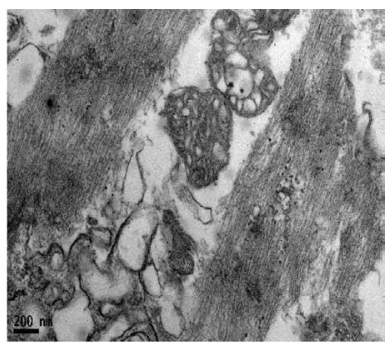

(c)

Figure 5. Effects of Cornus officinalis total glycoside on mitochondrial morphological changes after H/R injury. (a) Control group; (b) H/R group; (c) Low-dose group; (d) Middle-dose group; (e) High-dose group.

dependent signal transduction pathway [14]. Studies have shown that the increase of intracellular free calcium concentration in some pathological conditions can make a large number of $\mathrm{Ca}^{2+}$ accumulate in the mitochondria [15]. After the myocardial hypoxia/reoxygenation injury, the activity of $\mathrm{Ca}^{2+}$-ATPase in the cells changed, promoting $\mathrm{Ca}^{2+}$ to enter the cells, causing calcium overload. The increase of intracellular calcium induced the decrease of mitochondrial ATP formation, which further affects the metabolism of myocardial cells. At the same time, the increase of intracellular free calcium will activate calcium dependent proteolytic enzymes, such as phospholipase, protease, endonuclease, directly damage the plasma membrane, decompose cytoskeleton and nucleic acid, and cause the energy depletion of myocardial cells until irreversible damage. This study showed that H/R treatment caused mitochondrial dysfunction and cardiomyocyte apoptosis, whereas, pretreatment with Cornus officinalis Total Glycosides improved mitochondrial dysfunction and attenuated cell apoptosis.

Our results showed that the intracellular $\mathrm{Ca}^{2+}$ concentration increased significantly after treatment by hypoxia/reoxygenation, indicating that hypoxia/reoxygenation resulted in calcium overload. The possible mechanisms are as follows: when cardiomyocytes are anoxic, oxygen supply and oxidation substrates decrease, and then the function of ATP dependent myofibrils membrane calcium pump decreases, resulting in the decrease of $\mathrm{Ca}^{2+}$ scavenging ability and $\mathrm{Ca}^{2+}$ overload in cells [16]. In addition, hypoxia leads to the increase of $\mathrm{pH}$ in cardiac myocytes and the enhancement of $\mathrm{Na}^{+}-\mathrm{H}^{+}$exchange, which can reduce the degree of acidosis. However, the increase of intracellular $\mathrm{Na}^{+}$concentration can activate $\mathrm{Na}^{+}-\mathrm{Ca}^{2+}$ exchange, which in turn causes the increase of $\mathrm{Ca}^{2+}$ concentration in cells [17]. 
Intracellular calcium overload is the last common pathway of various damage factors. When $\mathrm{H} / \mathrm{R}$, both the inhibition of extracellular $\mathrm{Ca}^{2+}$ influx and the reduction of intracellular $\mathrm{Ca}^{2+}$ release can effectively reduce the occurrence of cardiomyocyte apoptosis [18]. Our study indicated that pretreatment with middle-dose and high-dose of Cornus officinalis Total Glycosides can block H/R-induced calcium overload of myocardial cells. By electron microscopy, we found that mitochondria were swollen, membrane ruptured when the myocardium was injured by hypoxia/reoxygenation. After pretreatment of Cornus officinalis Total Glycosides, the degree of myocardial injury induced by $\mathrm{H} / \mathrm{R}$ was significantly weakened and the mitochondrial membrane was intact in middle-dose and high-dose group.

The accumulation of $\mathrm{Ca}^{2+}$ in mitochondria enhances the permeability of the outer membrane of mitochondria, resulting in the increase of apoptosis factor in mitochondria, and further induces apoptosis. Middle-dose and high-dose of Cornus officinalis Total Glycosides could exert cardioprotective effects by inhibiting H/R-induced apoptosis of myocardial cells.

After H/R injury, the survival rate of myocardial cells decreased obviously, the rate of apoptosis increased evidently, the concentration of $\mathrm{Ca}^{2+}$ in cells increased, and the mitochondrial function of myocardial cells were destroyed. Based on the above results, we concluded that Cornus officinalis Total Glycosides pretreatment can inhibit cardiomyocyte apoptosis and calcium overload during H/R injury. However, the underlying mechanisms require us to further study.

\section{Source of Support}

This work was supported by the Guangdong province administration of traditional Chinese medicine (NO: 20181059).

\section{Conflicts of Interest}

The authors declare no conflicts of interest regarding the publication of this paper.

\section{References}

[1] Sun, L., Fan, H., Yang, L., Shi, L. and Liu, Y. (2015) Tyrosol Prevents Ischemia/Reperfusion-Induced Cardiac Injury in H9c2 Cells: Involvement of ROS, Hsp70, JNK and ERK, and Apoptosis. Molecules, 20, 3758-3775. https://doi.org/10.3390/molecules20033758

[2] Zhu, T., Yao, Q., Hu, X., Chen, C., Yao, H., Chao, J. (2015) The Role of MCPIP1 in Ischemia/Reperfusion Injury-Induced HUVEC Migration and Apoptosis. Cellular Physiology and Biochemistry, 37, 577-591. https://doi.org/10.1159/000430378

[3] Preda, M.B., Renningen, T., Burlacu, A., et al. (2014) Remote Transplantation of Mesenchymal Stem Cells Protects the Heart Against Is Chemia-Reperfusion Injury. Stem Cells, 27, 1002.

[4] Webster, K.A. (2012) Mitochondrial Membrane Permeabilization and Cell Death during Myocardial Infarction: Roles of Calcium and Reactive Oxygen Species. $\mathrm{Fu}$ ture Cardiology, 8, 863-884. https://doi.org/10.2217/fca.12.58 
[5] Shan, H., Yan, R., Diao, J., Lin, L., Wang, S., Zhang, M., Zhang, R. and Wei, J. (2015) Involvement of Caspases and Their Upstream Regulators in Myocardial Apoptosis in a Rat Model of Selenium Deficiency-Induced Dilated Cardiomyopathy. Journal of Trace Elements in Medicine and Biology, 31, 85-91. https://doi.org/10.1016/j.jtemb.2015.03.005

[6] Hadi, N.R., Al-Amran, F., Yousif, M., et al. (2013) Antiapoptotic Effect Ofsimvastatin Ameliorates Myocardial Ischemia/Reperfusion Injury. SRN Pharmacology, 2013, 19-26.

[7] Leone, T.C. and Kelly, D.P. (2011) Transcriptional Control of Cardiac Fuel Metabolism and Mitochondrial Function. Cold Spring Harbor Symposia on Quantitative Biology, 76, 175-182. https://doi.org/10.1101/sqb.2011.76.011965

[8] Chen, K., Li, J.J., Chen, K.F., Hou, X.P., Mai, H.C. and Xue, X.J. (2016) The Mechanism of Cornus officinalis Total Glycosides and Cornus Polysaccharide on Myocardial Protection in Rats with Acute Myocardial Infarction. Chinese Medicine, 7, 45-54. https://doi.org/10.4236/cm.2016.72007

[9] Xin, Y., Xu, X.F. and Huang, Y.M. (2011) Isolation Culture and Identification Neonatal Rat Cardiac Fibroblasts and Cardiac Myocytes. Journal of Xinxiang Medical University, 28, 541-547.

[10] Zeng, W.J., Liu, J.Y. and Ke, C.B. (2010) The Establishment of Hypoxia/Reoxygenation Injury of Rat Myocardial Cells by Liquid Paraffin Closure. Journal of Xunyang Medical College, 29, 108-110.

[11] Agrawal, P.B., Joshi, M., et al. (2012) Normal Myofibrillar Development Followed by Progressive Sarcomeric Disruption with Actin Accumulations in a Mouse Cfl2 Knockout Demonstrates Requirement of Cofilin-2 for Muscle Maintenance. Human Molecular Genetics, 21, 2341-2356. https://doi.org/10.1093/hmg/dds053

[12] Chen, K.F., Li, J.J., Pan, A.Z., et al. (2012) Effect of Cornus Officinalis Glycosideson Myocardiocyte Apoptosisin Suckling Mouse with Acute Hypoxia. Chinese Journal of Integrative Medicine on Cardio-Cerebrovascular Disease, 10, 1488-1489.

[13] Liakopoulos, O.J., Teucher, N., Muhlfeld, C., et al. (2006) Prevention of TNF Alpha Associated Myocardial Dysfunction Resulting from Cardiopulmonary Bypass and Cardioplegic Arrest by Glucocorticoid Treatment. European Journal of Cardio-Thoracic Surgery, 30, 263-270. https://doi.org/10.1016/j.ejcts.2006.04.019

[14] Ouyang, L., Shi, Z., Zhao, S., et al. (2012) Programmed Cell Death Pathways Incancer: A Review of Apoptosis, Autophagy and Programmed Necrosis. Cell Proliferation, 45, 487-498. https://doi.org/10.1111/j.1365-2184.2012.00845.x

[15] Navarro-Yepes, J., Burns, M., Anandhan, A., Khalimonchuk, O., del Razo, L.M., Quintanilla-Vega, B., Pappa, A., Panayiotidis, M.I. and Franco, R. (2014) Oxidative Stress, Redox Signaling, and Autophagy: Cell Death versus Survival. Antioxidants \& Redox Signaling, 21, 66-85. https://doi.org/10.1089/ars.2014.5837

[16] Wang, J.N., Qi, C., Liu, L.L., et al. (2018) Taurine Protects Primary Neonatal Car Pannax Quinquefolius Diomyocytes against Apoptosis Induced by Hydrogen Peroxide. International Heart Journal, 59, 190-196. https://doi.org/10.1536/ihj.16-372

[17] Bryant, S.M., Kong, C.H.T., Watson, J.J., et al. (2018) Caveolin 3-Dependent Loss of t-Tubular $\mathrm{I}_{\mathrm{Ca}}$ during Hypertrophy and Heart Failure in Mice. Experimental Physiology, 103, 652-665. https://doi.org/10.1113/EP086731

[18] Liu, Z., Zhang, F., Li, L.-Y., et al. (2014) Effect of Total Saponin of Pannax Quinquefolius on Cardiomyocyte Apoptosis Induced by Ischemia/Reperfusion and Calcium Concentration in Suckling Rats. Chinese Journal of Biologicals, 27, 661-670. 